\title{
QUELQUES OBSERVATIONS STATISTIQUES SUR LA VARIABLE ,NOMBRE DE SINISTRES” EN ASSURANCE AUTOMOBILE
}

\author{
P. THYRION \\ Bruxelles
}

\begin{abstract}
Résumé
Cette note ne risque pas de prêter le flanc à une critique souvent adressée à ASTIN, l'excès de spéculations mathématiques. Elle s'expose plutôt au reproche contraire, car elle a été, autant que possible, délibérément dépouillée de considérations théoriques, pour s'en tenir à quelques constatations arithmétiques fort simples sur la variable „,Nombre de sinistres”.

Ces constatations sont orientées vers la pratique, particulièrement vers la mise en œuvre d'un système de bonus-malus.
\end{abstract}

\section{INTRODUCTION}

Les observations présentées dans cette note prolongent celles qui ont été données dans une note antérieure [2] ${ }^{1}$ ) et qui couvraient un ensemble de I.4IO polices d'assurance pendant les années I955 à I958. Un sous-ensemble de I.094 polices a pu être observé jusqu'en I96I inclus, dans les conditions que nous rappelons ci-après.

Chaque police concerne un seul véhicule de la catégorie „Luxe” (équivalente à ,Tourisme et affaires"). Toutes ces polices ont été souscrites ou renouvelées dans le courant de l'année r954 et les véhicules correspondants ont donc été observés tous pendant les sept années civiles I955 à I96r.

Si un preneur d'assurance a changé de véhicule pendant la période considérée, l'observation a été continuée sur le nouveau véhicule. L'accent a donc été mis principalement sur le ou les conducteurs couverts par une police. Les sinistres pris en considération sont uniquement ceux qui ont donné lieu à un décaissement de la compagnie pour le risque de responsabilité civile du preneur d'assurance; les sinistres ,,sans suite" ont donc été exclus.

1) Les numéros entre crochets renvoient aux références indiquées in fine. 


\section{RESUltats STATISTIQUES PRINCIPAUX ${ }^{1}$ )}

a) Les nombres de véhicules $V_{n}$ ayant eu $n$ sinistres respectivement pendant $\mathrm{I}, 2, \ldots 7$ années consécutives sont donnés par le tableau I suivant.

Tableau I

\begin{tabular}{|c|c|c|c|c|c|c|c|}
\hline \multirow[b]{2}{*}{$n$} & \multicolumn{7}{|c|}{$V_{n}$} \\
\hline & I955 & $\begin{array}{l}\text { I955 } \\
\text { à } \\
\text { I956 }\end{array}$ & $\begin{array}{l}\text { I955 } \\
\text { à } \\
\text { I } 957\end{array}$ & $\begin{array}{l}\text { I955 } \\
\text { à } \\
\text { I958 }\end{array}$ & $\begin{array}{c}\text { I955 } \\
\text { à } \\
\text { I959 }\end{array}$ & $\begin{array}{l}\text { I955 } \\
\text { à } \\
\text { I } 960\end{array}$ & $\begin{array}{c}\text { I955 } \\
\text { à } \\
\text { I96r }\end{array}$ \\
\hline o & 937 & 823 & 723 & 619 & $54 \mathrm{I}$ & 472 & 423 \\
\hline I & I 34 & 202 & 249 & 288 & 298 & 306 & 306 \\
\hline 2 & I 8 & 46 & 86 & I I 7 & $\mathrm{r} 47$ & I 74 & I 84 \\
\hline 3 & 5 & I 8 & 20 & 39 & 55 & 65 & 77 \\
\hline 4 & & 3 & I I & 17 & 23 & 33 & 42 \\
\hline 5 & & 2 & 3 & 8 & I7 & 19 & I9 \\
\hline 6 & & & 2 & 2 & 6 & Io & 23 \\
\hline 7 & & & & 4 & I & 7 & 7 \\
\hline 8 & & & & & 3 & 2 & 5 \\
\hline 9 & & & & & 3 & 5 & 6 \\
\hline Io & & & & & & I & 2 \\
\hline
\end{tabular}

b) Les nombres de véhicules ayant eu respectivement $0,1,2, \ldots$ sinistres en I96r se ventilent comme suit en fonction du nombre de sinistres qu'ils ont eu, respectivement, au cours de l'année antérieure, des deux années antérieures, ..., des six années antérieures.

Tableau II

\begin{tabular}{r|r|r|r|r|r}
\hline I96I & 0 & I & 2 & 3 & 4 \\
\hline 1960 & & & & & \\
\hline 0 & 783 & IO5 & I3 & 2 & 2 \\
I & I19 & 32 & 4 & I & - \\
2 & I7 & 6 & 4 & 2 & - \\
3 & I & I & - & - & I \\
4 & - & - & - & - & - \\
5 & I & - & - & - & - \\
\hline
\end{tabular}

1) Je remercie vivement M. Georges Martin, Président-Directeur Général de la Royale Belge, qui m'a autorisé à faire établir et à exploiter ces statistiques. 
„NOMBRE DE SINISTRES" EN ASSURANCE AUTOMOBILE 205

TABleaU III

\begin{tabular}{|c|c|c|c|c|c|}
\hline \multicolumn{6}{|c|}{1961} \\
\hline $\begin{array}{l}1959 \\
190 \\
\text { à } 1960 \\
\end{array}$ & 0 & I & 2 & 3 & 4 \\
\hline o & 673 & 78 & 10 & I & 2 \\
\hline I & 178 & 49 & 5 & I & 一 \\
\hline 2 & $5^{2}$ & IO & 5 & 3 & - \\
\hline 3 & I I & 4 & - & - & - \\
\hline 4 & 6 & 2 & $\mathbf{I}$ & - & - \\
\hline 5 & I & I & - & 一 & I \\
\hline
\end{tabular}

TABLEAU IV

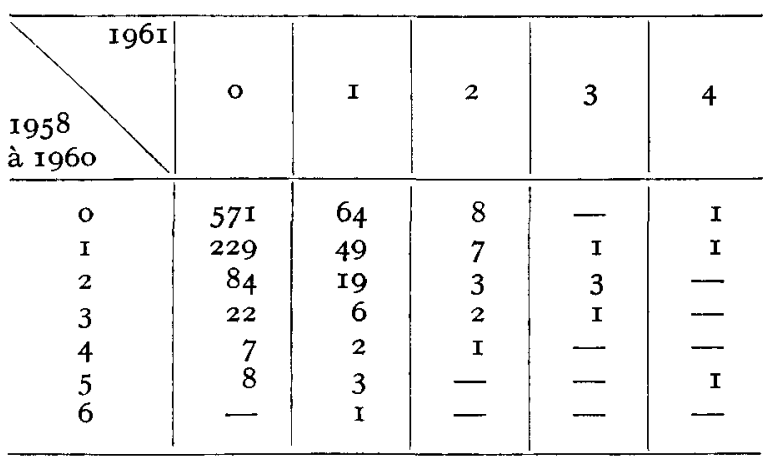

Tableau V

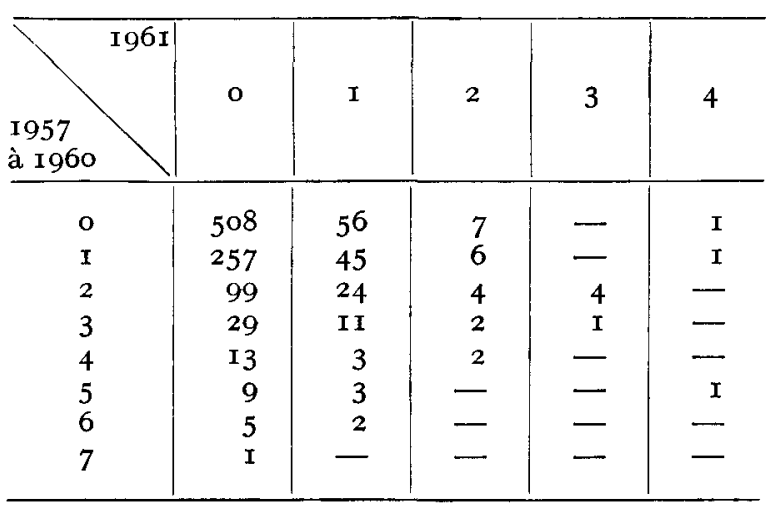


Tabieau VI

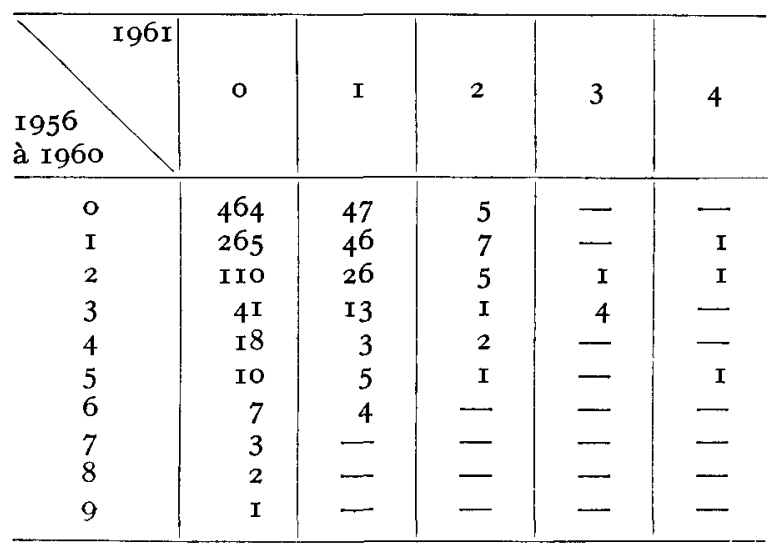

TABLEAU VII

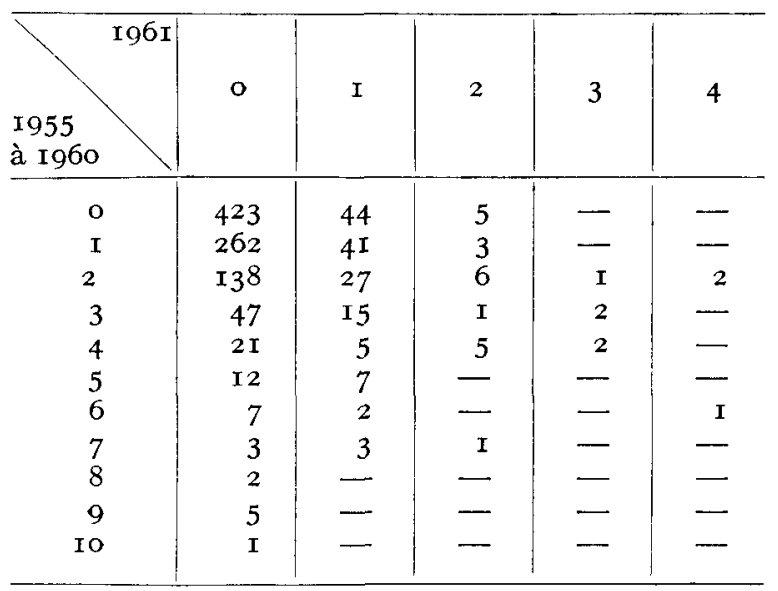

Des ventilations analogues pour chacun des exercices de 1960 à I956 par rapport aux précédents ont également été établies. Nous n'en donnons pas le détail pour ne pas allonger la note.

\section{Analyse de ces statistigues}

De nombreux essais de compensation de la loi de probabilité du nombre de sinistres par une loi de Poisson composée ont déjà été effectués. Notre objectif n'est pas d'en refaire sur la base des 
statistiques indiquées ci-dessus Nous nous proposons plutôt de tester par des voies indirectes la validité d'un tel schéma, en exploitant le fait que ces statistiques portent sur une assez longue période.

Ier test

Si la loi de probabilité du nombre de sinistres $N(t)$ est

$$
P(n ; t)=\int_{0}^{\infty} e^{-\lambda p t} \frac{(\lambda p t)^{n}}{n !} d U(\lambda)
$$

on a

$$
\begin{aligned}
E[N(t)] & =\sum_{n=1}^{-\infty} n \int_{0}^{\infty} e^{-\lambda p t} \frac{(\lambda p t)^{n}}{n !} d U(\lambda) \\
& =p t \int_{0}^{\infty} \lambda d U(\lambda) e^{-\lambda p t} \sum_{n=1}^{-\infty} \frac{(\lambda p t)^{n-1}}{(n-I) !}= \\
& =p t E(\Lambda)
\end{aligned}
$$

De même

$E\left[N^{2}(t)\right]=p t E(\Lambda)+p^{2} t^{2} E\left(\Lambda^{2}\right)$

$E\left[N^{3}(t)\right]=p t E(\Lambda)+3 p^{2} t^{2} E\left(\Lambda^{2}\right)+p^{3} t^{3}\left(\Lambda^{3}\right)$

Comme Seal l'a observé [I], si la fonction de structure $U(\lambda)$ est invariable dans le temps, on doit avoir quel que soit $t$

$$
\frac{E\left[N^{2}(t)\right]-E[N(t)]}{E^{2}[N(t)]}=\frac{E\left(\Lambda^{2}\right)}{E^{2}(\Lambda)}=\text { constante }
$$

Prolongeant cette idée, on peut ajouter que l'on a également, entre autres,

$$
\frac{E\left[N^{3}(t)\right]-3 E\left[N^{2}(t)\right]+2 E[N(t)]}{E^{3}[N(t)]}=\frac{E\left(\Lambda^{3}\right)}{E^{3}(\Lambda)}=\text { constante }
$$

Remarquons que si le taux instantané de sinistre $p$ n'était pas constant, on remplacerait $p t$ par $\int_{0}^{t} p(u)$ du et les relations (I) et (2) se maintiendraient. 
L'application de ces formules aux observations statistiques du tableau I conduit aux résultats suivants:

\begin{tabular}{c|c|c|c|c|c|c|c}
\hline & I955 & $\begin{array}{c}\text { I955 à } \\
\text { I956 }\end{array}$ & $\begin{array}{c}\text { I955 à } \\
\text { I957 }\end{array}$ & $\begin{array}{c}\text { I955 à } \\
\text { I958 }\end{array}$ & $\begin{array}{c}\text { I955 à } \\
\text { I959 }\end{array}$ & $\begin{array}{c}\text { I955 à } \\
\text { I960 }\end{array}$ & $\begin{array}{c}\text { I955 à } \\
\text { I96I I }\end{array}$ \\
\hline (I) & 2, I I & 2,20 & 1,95 & I,87 & I,9I & I,85 & I,82 \\
$(2)$ & 5,67 & 7,09 & 5,72 & $5,4 \mathrm{I}$ & 5,90 & 5,38 & 4,90 \\
\hline
\end{tabular}

On ne pouvait évidemment espérer une constance parfaite de résultats portant sur des nombres de sinistres faibles et faisant intervenir en outre des moments du $2 \mathrm{e}$ et du $3 \mathrm{e}$ ordre. Néanmoins, malgré les irrégularités il nous semble que les résultats ci-dessus n'infirment pas la vraisemblance d'un schéma basé sur l'invariabilité dans le temps de la fonction de structure.

Si l'on se rapporte à la médiane de chaque série soit

$$
\begin{aligned}
& \frac{E\left(\Lambda^{2}\right)}{E^{2}(\Lambda)}=\mathrm{I}, 9 \mathrm{I} \\
& \frac{E\left(\Lambda^{3}\right)}{E^{3}(\Lambda)}=5.67
\end{aligned}
$$

on voit que la fonction de structure $U(\lambda)$ est du type hypoexponentiel, c'est-à-dire plus concentrée que la loi exponentielle pour laquelle

$$
\frac{E\left(\Lambda^{2}\right)}{E^{2}(\Lambda)}=2 \quad \text { et } \frac{E\left(\Lambda^{3}\right)}{E^{3}(\Lambda)}=6
$$

Ce pourrait donc être une loi avec un seul mode situé près de l'origine.

2 ème test

Si la loi de probabilité du nombre de sinistres est du type Poisson composé, l'hétérogénéité de l'ensemble des risques considérés doit apparaître si l'on subdivise cet ensemble en sous-ensembles définis par le nombre de sinistres observés pendant une période antérieure. Sur la base des tableaux II à VII et analogues, nous avons donc calculé le rapport entre la valeur moyenne du nombre de sinistres par véhicule-année pour ces sous-ensembles et la valeur moyenne du 
même nombre pour l'ensemble des risques, et ce pour chacune des années de 1956 à I96I analysée en fonction du nombre $n$ de sinistres pendant les périodes précédentes de I à 6 ans selon les cas. Voici les résultats groupés - pour la facilité des comparaisons - selon le nombre d'années précédentes définissant les sous-ensembles considérés.

\begin{tabular}{c|c|c|c|c|c|c}
\hline $\begin{array}{c}n \\
\text { pendant I' } \\
\text { année précédente }\end{array}$ & I96I & 1960 & I959 & I958 & I957 & I956 \\
\hline o & 0,82 & 0,85 & 0,86 & 0,88 & $0,9 \mathrm{I}$ & $0,8 \mathrm{I}$ \\
$\mathrm{I}$ & $\mathrm{I}, 42$ & $\mathrm{I}, 46$ & $\mathrm{I}, 54$ & $\mathrm{I}, 5 \mathrm{I}$ & $\mathrm{I}, 45$ & $\mathrm{I}, 99$ \\
2 & 3,54 & 2,58 & $\mathrm{I}, 78$ & 3,26 & $2, \mathrm{I} 5$ & 2,63 \\
3 & 6,42 & 3,20 & 3,65 & 0 & 2,40 & 4,73 \\
\hline
\end{tabular}

\begin{tabular}{|c|c|c|c|c|c|c|c|c|}
\hline $\begin{array}{c}n \\
\text { pendant les } \\
2 \text { années précédentes }\end{array}$ & \multicolumn{2}{|c|}{ I96I } & \multicolumn{2}{|c|}{ I960 } & \multicolumn{2}{|c|}{ I959 } & \multicolumn{2}{|c|}{$195^{8}$} \\
\hline$o$ & \multicolumn{2}{|c|}{0,73} & \multicolumn{2}{|c|}{0,79} & \multicolumn{2}{|c|}{0,78} & \multicolumn{2}{|c|}{0,79} \\
\hline I & \multicolumn{2}{|c|}{$\mathbf{I}, 37$} & \multicolumn{2}{|c|}{1,22} & \multicolumn{2}{|c|}{$\mathrm{I}, 27$} & \multicolumn{2}{|c|}{$\mathrm{I}, 25$} \\
\hline 2 & \multicolumn{2}{|c|}{2,13} & \multicolumn{2}{|c|}{ I,99 } & \multicolumn{2}{|c|}{2,43} & \multicolumn{2}{|c|}{2,37} \\
\hline$\geqslant 3$ & \multicolumn{2}{|c|}{2,47} & \multicolumn{2}{|c|}{2,57} & \multicolumn{2}{|c|}{2,27} & \multicolumn{2}{|c|}{3,99} \\
\hline $\begin{array}{c}n \\
\text { pendant les } \\
3 \text { années précéder }\end{array}$ & & \multicolumn{2}{|c|}{ I96I } & \multicolumn{2}{|c|}{1960} & & 59 & \\
\hline 0 & & \multicolumn{2}{|c|}{0,67} & \multicolumn{2}{|c|}{0,73} & \multicolumn{2}{|c|}{0,69} & \\
\hline I & & \multirow{2}{*}{\multicolumn{2}{|c|}{$\begin{array}{l}1,25 \\
160\end{array}$}} & \multicolumn{2}{|c|}{$I, 20$} & \multicolumn{2}{|c|}{$\mathrm{I}, \mathrm{I} 5$} & \\
\hline 2 & & & & & & & & \\
\hline$\geqslant 3$ & & \multicolumn{2}{|c|}{2,28} & & & \multicolumn{2}{|c|}{2,70} & \\
\hline \multicolumn{3}{|c|}{$\begin{array}{c}n \\
\text { pendant les } \\
4 \text { années précédentes }\end{array}$} & \multicolumn{2}{|c|}{ I96I } & \multicolumn{2}{|c|}{ I960 } & \multicolumn{2}{|c|}{ I959 } \\
\hline \multicolumn{3}{|l|}{0} & & & & & & \\
\hline I & & & & & & & & \\
\hline 2 & & & & & & & & \\
\hline 3 & & & & & & & & \\
\hline$\geqslant 4$ & & & & & & & & \\
\hline
\end{tabular}




\begin{tabular}{c|c|c}
\hline $\begin{array}{c}n \\
\text { pendant les } \\
\text { 5 années précédentes }\end{array}$ & I96I & I960 \\
\hline 0 & 0,57 & 0,72 \\
$\mathrm{I}$ & $\mathrm{I}, 03$ & 0,89 \\
2 & $\mathrm{I}, 54$ & $\mathrm{I}, 2 \mathrm{I}$ \\
3 & 2,35 & 2,09 \\
$\geqslant 4$ & $\mathrm{I}, 98$ & $2,8 \mathrm{I}$ \\
\hline
\end{tabular}

\begin{tabular}{c|c}
$\begin{array}{c}n \\
\text { pendant les } \\
6 \text { années précédentes }\end{array}$ & I96I \\
\hline o & 0,59 \\
I & 0,79 \\
2 & 1,48 \\
3 & 1,82 \\
$\geqslant 4$ & 2,60 \\
\hline
\end{tabular}

L'échelonnement des valeurs de ce rapport répond assez bien aux prévisions théoriques qui seraient basées sur une loi de Poisson composée et ce quelle que soit l'année considérée. Cela apparaît encore mieux si, pour niveler les anomalies que le faible volume de certains sous-ensembles ne peut manquer de provoquer, on ne retient que la médiane (ou éventuellement la moyenne des médianes) de chaque ligne, ce qui conduit au tableau suivant.

\begin{tabular}{|c|c|c|c|c|c|c|}
\hline \multirow[b]{2}{*}{$n$} & \multicolumn{6}{|c|}{ Valeurs médianes du rapport s'il y a eu $n$ sinistres pendant } \\
\hline & $\begin{array}{l}\text { I'année } \\
\text { précédente }\end{array}$ & $\begin{array}{l}\text { les } 2 \text { années } \\
\text { précédentes }\end{array}$ & $\begin{array}{l}\text { les } 3 \text { années } \\
\text { précédentes }\end{array}$ & $\begin{array}{l}\text { les } 4 \text { années } \\
\text { précédentes }\end{array}$ & $\begin{array}{l}\text { les } 5 \text { années } \\
\text { précédentes }\end{array}$ & $\begin{array}{l}\text { les } 6 \text { années } \\
\text { précédentes }\end{array}$ \\
\hline o & 0,85 & 0,79 & $0,7 \mathrm{I}$ & 0,66 & 0,64 & 0,59 \\
\hline I & $\mathrm{I}, 48$ & $\mathrm{I}, 25$ & $\mathrm{I}, 2 \mathrm{I}$ & $\mathrm{I}, \mathrm{OI}$ & 0,96 & 0,79 \\
\hline 2 & $2,6 \mathrm{I}$ & 2,13 & $I, 59$ & $\mathrm{I}, 7 \mathrm{I}$ & 1,38 & $\mathrm{I}, 4^{8}$ \\
\hline$\geqslant 3$ & $3,4 \mathrm{I}$ & 2,57 & 2,66 & & & \\
\hline 3 & & & & 2,07 & 2,22 & 1,82 \\
\hline$\geqslant 4$ & & & & 2,98 & 2,39 & 2,60 \\
\hline
\end{tabular}

\section{Conclusions}

Le seul mérite des statistiques utilisées - si elles en ont un - est la relative longueur de la période d'observation qui compense, dans une certaine mesure, le trop petit nombre d'unités observées. A défaut de pouvoir y appliquer des tests statistiques précis, on a pu suivre le phénomène dans le temps et s'assurer notamment d'une stabilité approximative de la fonction de structure $U(\lambda)$.

On peut en conclure, avec une vraisemblance suffisante pour la pratique nous semble-t-il, que l'hypothèse de l'hétérogénéité des classes de risque se montre apte à prévoir l'influence du comportement passé d'une sous-classe de risques sur son comportement futur en ce qui concerne le nombre de sinistres. 
La mesure de cette influence est une des bases pratiques d'un système de bonus-malus qui introduirait a posteriori dans les tarifs l'effet du facteur humain sur l'intensité du risque, sous réserve de pouvoir admettre l'indépendance stochastique des variables „Nombre de sinistres" et „Coût d'un sinistre”.

\section{BIBLIOGRAPHIE}

[I] H. L. Seal: Stochastic Theory of a Risk Business (p. 25).

[2] P. Thyrion: Etude de la loi de probabilité de la variable „Nombre de sinistres" dans l'assurance automobile - XVIe Congrès International d'Actuaires Ig6o. 